\title{
Frozen sections are unreliable for the diagnosis of necrotizing soft tissue infections
}

\author{
Isaac H Solomon ${ }^{1}$, Rene Borscheid ${ }^{2,3}$, Alvaro C Laga ${ }^{1}$, Reza Askari ${ }^{2}$ and Scott R Granter ${ }^{1}$ \\ ${ }^{1}$ Department of Pathology, Brigham and Women's Hospital, Harvard Medical School, Boston, MA, USA and \\ ${ }^{2}$ Department of Surgery, Brigham and Women's Hospital, Harvard Medical School, Boston, MA, USA
}

\begin{abstract}
Necrotizing soft tissue infections are rare but are associated with high rates of morbidity and mortality. The use of bedside or intraoperative frozen sections has been reported to be associated with faster diagnosis and better outcomes; however, to date no large studies have been published to determine the sensitivity and specificity of frozen sections in this setting. Twenty years of cases suspicious for necrotizing soft tissue infection at a large academic referral center were reviewed, blinded to the final clinical diagnosis (gold standard). Cases were assessed for the number of neutrophils, extent of necrosis, presence of thrombi, bacteria, karyorrhexis, and fibrin, and concordance with permanent sections. A total of 166 cases suspicious for necrotizing soft tissue infection had frozen section slides available for review. Sixty-three cases were clinically determined to be positive and 103 negative. Neutrophils, necrosis, thrombi, bacteria, karyorrhexis, and fibrin were present in both positive and negative cases; however, no histological feature or combination of features was found to be both sensitive and specific for necrotizing soft tissue infection. The combined presence of necrosis and frequent neutrophils was $73 \%$ sensitive and $68 \%$ specific, with a $58 \%$ positive predictive value and $80 \%$ negative predictive value. The additional observation of bacteria decreased sensitivity to $32 \%$, whereas raising specificity to $91 \%$, with $69 \%$ positive predictive value and $68 \%$ negative predictive value. Thirty-two cases $(19 \%)$ contained findings identified on permanent sections (eg, bacteria) not observed on frozen section slides, highlighting the risk of false negatives owing to technical limitations or sampling errors. Frozen sections in necrotizing soft tissue infections and negative cases may show similar histological findings. Combined with the risk of false negatives, these results suggest that frozen sections are likely to be of limited clinical utility due to lack of sensitivity and specificity, and risk for delayed diagnosis and treatment.

Modern Pathology (2018) 31, 546-552; doi:10.1038/modpathol.2017.173; published online 15 December 2017
\end{abstract}

Necrotizing soft tissue infections, including necrotizing cellulitis, necrotizing fasciitis, Fournier's gangrene, and necrotizing myositis, are aggressive soft tissue infections associated with significant morbidity and mortality. ${ }^{1,2}$ Even with optimal treatment, consisting of radical surgical debridement and broad spectrum antibiotics, mortality approaches $20 \% .^{3-5}$ The majority of necrotizing soft tissue infections are polymicrobial (70-90\%), caused by aerobic and anaerobic bacteria from the gastrointestinal and genitourinary tracts. ${ }^{6}$ Polymicrobial infections typically involve the trunk or perineum and are

Correspondence: SR Granter, MD, Associate Pathologist, Brigham and Women's Hospital, and Associate Professor of Pathology, Harvard Medical School, 75 Francis Street, Boston, MA 02115, USA.

E-mail: sgranter@bwh.harvard.edu

${ }^{3}$ Current address: Department of Surgery, Houston Methodist Hospital, Houston, TX, USA.

Received 30 August 2017; revised 14 October 2017; accepted 15 October 2017; published online 15 December 2017 associated with perianal abscesses, bowel surgery, decubitus ulcers, drug injection sites, and spread from genital sites. Monomicrobial infections, including Streptococcus pyogenes, Staphylococcus aureus, Vibrio spp., Aeromonas spp., and other Gramnegative and anaerobic bacteria, typically involve the extremities and are associated with diabetes, cardiovascular disease, ulcers, injected drug use, varicella, and minor injuries. ${ }^{7}$

Necrotizing soft tissue infections can be difficult to diagnose early in the disease course due to similarities with more superficial infections including cellulitis and erysipelas. Clinical signs and symptoms include severe, seemingly disproportionate pain, failure to respond to initial antibiotics, systemic toxicity with altered mental status, edema, bullous lesions, gas or crepitus, skin necrosis, and ecchymosis. ${ }^{8}$ Immunocompromised patients present additional challenges owing to lack of typical features at presentation, resulting in delayed diagnosis and increased mortality. ${ }^{9}$ Utilization of common laboratory values (ie, C-reactive protein, white 
blood cell count, hemoglobin, sodium, creatinine, and glucose) to calculate the Laboratory Risk Indicator for Necrotizing Fasciitis (LRINEC) score has been extensively studied, but may have limited sensitivity in some settings. ${ }^{10-12}$ A variety of imaging modalities have been evaluated including X-ray, magnetic resonance imaging, computed tomography, and ultrasound, which can show deep fascial thickening, enhancement, fluid and gas in the soft tissue plane, including the superficial fascia. ${ }^{13,14}$ Gross inspection can be conducted in the operating room or at the bedside using the 'finger test', a procedure in which a $2-\mathrm{cm}$ incision is made into the deep fascia. Positive findings include gray necrotic fascia, foul smelling 'dishwater' fluid, lack of bleeding, and lack of resistance to blunt dissection. ${ }^{15,16}$

Histological findings associated with necrotizing soft tissue infection include necrosis of the superficial fascia, neutrophilic infiltration, fibrin thrombi within blood vessels, and the presence of microorganisms. Intraoperative frozen sections have been used in this setting, first described by Stamenkovic et al. ${ }^{17}$ in 1984, and were proposed to be associated with earlier diagnosis and better outcomes. Subsequent studies have shown that frozen sections are reasonably sensitive for the diagnosis of necrotizing soft tissue infection, although these conclusions are based on a total of 34 positive cases. ${ }^{18-20}$ The goal of this study was to assess the utility and reliability of frozen sections for the diagnosis of necrotizing soft tissue infection via retrospective review of cases over a 20 -year period at a large academic medical center.

\section{Materials and methods}

Following Institutional Review Board approval, cases at the Brigham and Women's Hospital from 1995 to 2015 were identified for which a bedside or intraoperative frozen section was performed for initial diagnosis in cases suspicious for a necrotizing soft tissue infection. Frozen sections from recurrent debridements or for evaluation of margins with an established diagnosis of necrotizing soft tissue infection were excluded from this study. The three study pathologists independently reviewed all cases blinded to final clinical diagnosis. Hematoxylin and eosin-stained frozen section slides and formalinfixed paraffin-embedded remnants were examined for each case. Slides were evaluated for the following features: number of neutrophils per high power field (0, 1-5, 6-24, >25); extent of necrosis (absent, equivocal, focal, extensive $>25 \%$ ); presence of thrombi, bacteria, karyorrhexis, and fibrin; and concordance with permanent sections including Gram stains when available. The histological features were compared with the final clinical diagnosis (considered to be the gold standard) and culture results. Clinical diagnosis was determined by agreement of two specialists in critical care surgery after review of all available information in the electronic medical records, including operative notes, clinical course, and other laboratory, radiological, and clinical findings. Statistics were calculated by Student's $t$-test or $\chi^{2}$ with $P<0.05$ considered significant. Kappa statistics for interobserver variability were calculated using STATA (StataCorp, College Station, TX, USA).

\section{Results}

From 1995 to 2015, 175 surgical pathology cases were identified that included at least one frozen section to diagnose or rule out a necrotizing soft tissue infection. In the vast majority of cases, the frozen section and final pathologic diagnoses were descriptive, with the occasional note that the findings would be consistent with a diagnosis of necrotizing soft tissue infection in the appropriate clinical setting. A total of 166 cases were available for review, including 63 positive and 103 negative cases, categorized by retrospective chart review for final clinical diagnosis. An average of three necrotizing soft tissue infections (range 0-7) and five negative cases (range 0-12) per year were reported, with a slight peak in 2002 followed by a steady decline in total number of biopsies. A power analysis was performed which calculated a power of 0.88 to determine a difference between the necrotizing soft tissue infection-positive and negative cases. The median ages were similar between the two groups (54 vs 52 years, $P=0.8$ ) with a similar sex distribution (52 vs $50 \%$ male, $P=0.7$ ). Limbs were the most common site $(61 \%$; 36 positive and 65 negative cases), followed by trunk ( $27 \% ; 18$ and 26 cases), and perineum ( $7 \% ; 8$ and 4 cases), whereas no site was specified in the remaining cases ( $5 \%, 1$ and 8 cases).

Histological review of frozen sections frequently identified features associated with infection and inflammation in both the necrotizing soft tissue infection-positive and negative cases (Table 1, Figure 1). Although a greater proportion of necrotizing soft tissue infection-positive cases contained neutrophils than negative cases $(P=0.0003),>25$ neutrophils per high power field was a common finding in both positive $(79 \%)$ and negative $(46 \%)$ cases. In contrast, negative cases were more commonly associated with lack of neutrophils (24\%) than positive cases $(6 \%)$. Similarly, extensive necrosis $(>25 \%$ of tissue) was present in both positive $(51 \%)$ and negative $(12 \%)$ cases, but more common in necrotizing soft tissue infection-positive cases $(P<0.0001)$, and lack of necrosis was more frequently observed in negative $(63 \%)$ than positive cases $(24 \%)$. Fibrin thrombi were rarely identified in either positive or negative cases, with no significant difference in frequency between groups. Bacteria (37 vs 9\%; $P<0.0001)$, karyorrhexis (71 vs $32 \% ; P<0.0001$ ), and fibrin ( 71 vs $44 \% ; P=0.005$ ) were all present more frequently in necrotizing soft tissue infection-positive than negative cases. Positive predictive values and 
Table 1 Histological features of necrotizing soft tissue infection-positive and negative cases

\begin{tabular}{|c|c|c|c|c|c|c|}
\hline Characteristic & Result & $\begin{array}{l}\text { Necrotizing soft } \\
\text { tissue infection- } \\
\text { positive }(\mathrm{n}=63)\end{array}$ & $\begin{array}{l}\text { Necrotizing soft tissue } \\
\text { infection-negative } \\
(\mathrm{n}=103)\end{array}$ & P-value & $\begin{array}{l}\text { Positive } \\
\text { predictive } \\
\text { value }\end{array}$ & $\begin{array}{l}\text { Negative } \\
\text { predictive } \\
\text { value }\end{array}$ \\
\hline \multirow[t]{4}{*}{ Neutrophils (per high power field) } & 0 & $4(6)$ & $25(24)$ & & & \\
\hline & $1-5$ & $4(6)$ & $16(16)$ & & & \\
\hline & $6-24$ & $5(8)$ & $15(15)$ & & & \\
\hline & $>25$ & $50(79)$ & 47 (46) & 0.0003 & 47 & 84 \\
\hline \multirow[t]{4}{*}{ Necrosis (extent) } & Absent & $15(24)$ & $65(63)$ & & & \\
\hline & Equivocal & $1(2)$ & $4(4)$ & & & \\
\hline & Focal & $15(24)$ & $22(21)$ & & & \\
\hline & $\begin{array}{c}\text { Extensive } \\
(>25 \%)\end{array}$ & $32(51)$ & $12(12)$ & $<\mathbf{0 . 0 0 0 1}$ & 58 & 81 \\
\hline \multirow[t]{2}{*}{ Thrombi } & Absent & $60(95)$ & $100(97)$ & & & \\
\hline & Present & $3(5)$ & 3 (3) & 0.5 & 38 & 42 \\
\hline \multirow[t]{2}{*}{ Bacteria } & Absent & $40(64)$ & $94(91)$ & & & \\
\hline & Present & $23(37)$ & $9(9)$ & $<0.0001$ & 72 & 70 \\
\hline \multirow[t]{2}{*}{ Karyorrhexis } & Absent & $18(29)$ & $70(68)$ & & & \\
\hline & Present & $45(71)$ & 33 (32) & $<0.0001$ & 58 & 80 \\
\hline \multirow{2}{*}{ Fibrin } & Absent & $18(29)$ & $58(56)$ & & & \\
\hline & Present & $45(71)$ & $45(44)$ & 0.0005 & 50 & 76 \\
\hline
\end{tabular}

Values represent number (percentage). $P$-values from Student's $t$-test; $<0.05$ (bold) interpreted as significant. For positive and negative predictive value calculations, $6-24$ or $>25$ neutrophils per high power field and focal or extensive ( $>25 \%)$ necrosis were considered positive values.

negative predictive values are listed in Table 1 for each histological feature. No individual feature or combination of histological features was found to be both sensitive and specific for necrotizing soft tissue infection. For example, the presence of neutrophils (6-24 or $>25 / \mathrm{hpf}$ ) and necrosis (focal or extensive) was $73 \%$ sensitive and $68 \%$ specific, with a $58 \%$ positive predictive value and $80 \%$ negative predictive value. The additional observation of bacteria decreased sensitivity to $32 \%$, whereas raising specificity to $91 \%$, with $69 \%$ positive predictive value and $68 \%$ negative predictive value.

Final culture results were reviewed from all necrotizing soft tissue infection-positive cases (Table 2). A small but not statistically significant increase in culture positivity was identified when organisms were observed histologically (96 vs 83\%; $P=0.13$ ). Polymicrobial infections (average of 4.2 organisms isolated per culture) were present in 18/63 $(29 \%)$ cases, including seven with organisms seen on hematoxylin and eosin frozen sections. Group A beta-hemolytic streptococci (ie, S. pyogenes), was the most common monomicrobial isolate $(n=15)$, followed by $S$. aureus $(\mathrm{n}=6)$, and coagulase-negative staphylococci $(n=4)$. The remaining cultures included 1-2 cases each of Group G streptococci, S. pneumoniae, Clostridium septicum, Escherichia coli, Pseudomonas aeruginosa, Serratia marcescens, and Candida spp. Negative cultures were associated with one case that contained histologically observable organisms, and seven cases with no definitive bacteria.

Permanent section hematoxylin and eosin slides were available for review in 163 cases. Gram stains were performed on permanent sections in $67 / 166$ $(40 \%)$ cases, and detected bacteria in 17/28 (61\%) necrotizing soft tissue infection and 7/39 (18\%) negative cases. Comparison of the histological findings in frozen and permanent sections showed discordance in at least one histological feature in 32 cases, including 18 necrotizing soft tissue infection-positive and 14 negative cases. The most frequent discordant feature was lack of bacteria observed on frozen section slides in 23 cases, with organisms detected on permanent section hematoxylin and eosin slides $(n=11)$ or Gram stains $(n=12)$. Other features absent on frozen but observed on permanent section slides included lack of fibrin thrombi $(n=5)$, lack of necrosis $(n=3)$, lack of or fewer neutrophils $(\mathrm{n}=2)$, and lack of fungal hyphae $(n=1)$. Overall, concordance was decreased for frozen sections compared to permanent sections for necrotizing soft tissue infection-positive and negative cases (71 vs $86 \% ; P=0.02$ ). Independent review of all cases blinded to the final diagnosis produced substantial variations in the evaluation of histological features between the three study pathologists (Table 3). Complete concordance was seen in $60 \%$ of observations overall, with the presence of fibrin thrombi $(77 \%)$ and bacteria $(73 \%)$ being the most commonly agreed upon features. A minority of cases contained no agreement for number of neutrophils $(12 \%)$ or extent of necrosis $(16 \%)$. Kappa statistics for interobserver variability were substantial for thrombi (0.70), moderate for necrosis (0.41), bacteria (0.43), and fibrin (0.45), and fair for neutrophils (0.39) and karyorrhexis (0.32). Each pair of pathologists exhibited similar levels of agreement (69-75\%).

\section{Discussion}

In this study at a large academic medical center, 166 cases with frozen sections were reviewed from 

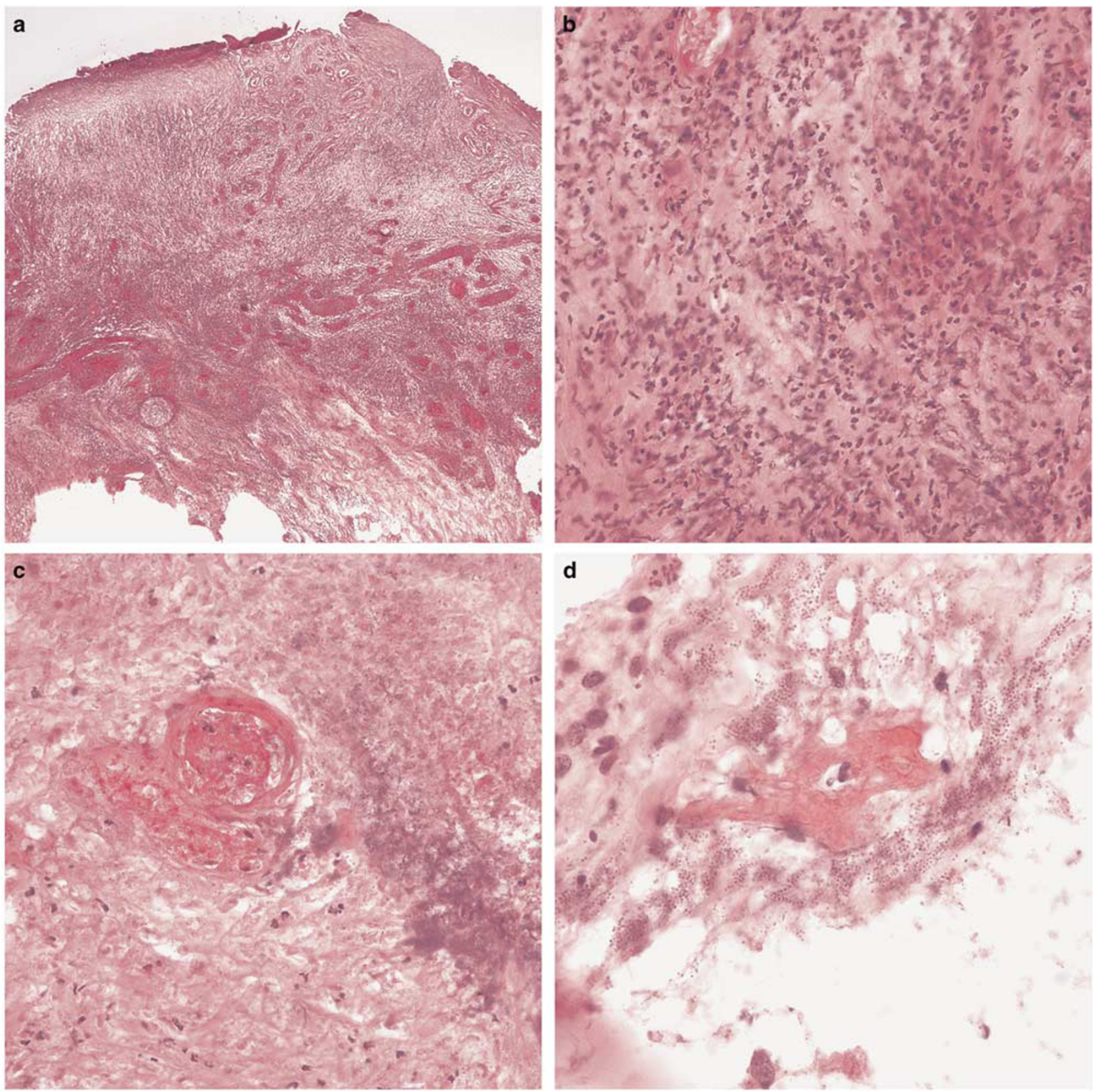

Figure 1 Histological features observed in frozen section biopsies of necrotizing soft tissue infections. Low-magnification view of a fascial biopsy from a case of necrotizing soft tissue infection shows large areas of necrosis. (a) Higher magnification images demonstrate the presence of abundant neutrophils (b), blood vessels with fibrin thrombi (c), and bacteria (d). Low (e) and high-magnification (f) images from a negative case show similar findings of necrosis and abundant neutrophils.

patients suspicious for a necrotizing soft tissue infection. The major histological findings present on routine histology with formalin-fixed paraffinembedded tissue, including necrosis, acute inflammation, bacteria, thrombi, karyorrhexis, and fibrin were all present in frozen section slides from cases positive for necrotizing soft tissue infection. All of the abnormalities observed in necrotizing soft tissue infection cases were also present, but less frequently, in negative cases, with the exception of thrombi which were identified in a small minority of cases
$(<5 \%$ ). However, no individual histological feature was pathognomonic for necrotizing soft tissue infection, and no combination of findings was both sensitive and specific.

To further investigate the reliability of frozen sections, the corresponding permanent sections for each case were subsequently reviewed, which identified histological features absent on frozen section slides in 32 cases (19\%). The most common discordant feature was the presence of bacteria, which can be difficult to visualize depending on 

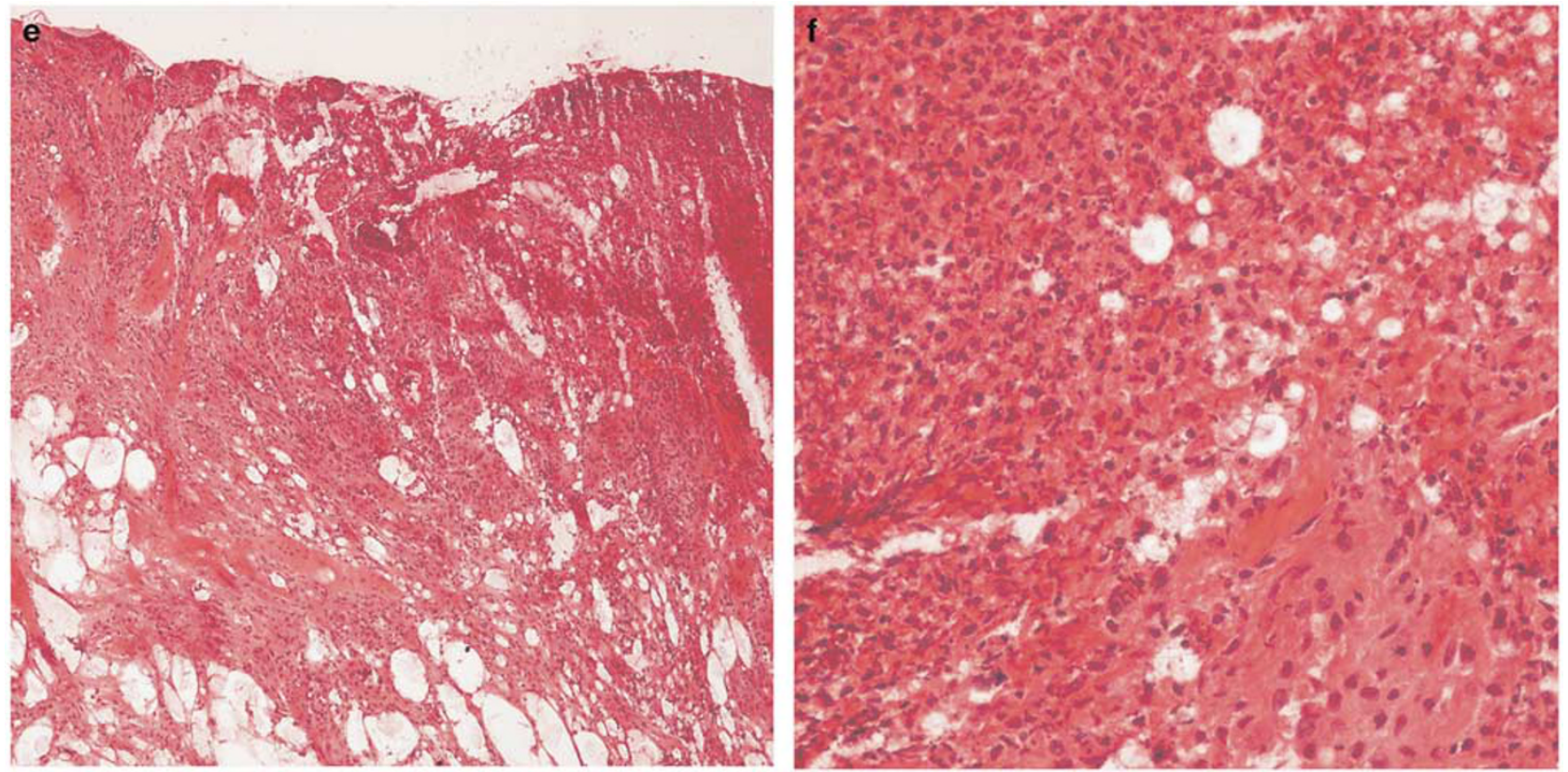

Figure 1 (Continued)

the quality and thickness of the frozen section slides, the quality of the hematoxylin and eosin staining including interference with hematoxylin pigment deposition, and the quantity, density, and type of bacteria. Thrombi, necrosis, and neutrophils may also be falsely negative on frozen sections due to similar artifacts, but are more likely to be negative due to sampling errors. These errors may occur when the specimen received for evaluation is too large to entirely freeze and non-diagnostic tissue is selected, or when the diagnostic tissue is unevenly distributed in the block and is not represented on the frozen section slides. ${ }^{21}$ Sampling errors and artifacts cannot be totally eliminated, but can be minimized by experienced pathologists who interpret a high volume of frozen sections. ${ }^{22}$ The equivocal presence of bacteria could be resolved by an intraoperative request for a STAT Gram stain from the clinical microbiology laboratory. Although this practice has not proven to be beneficial in revision spinal surgery or revision total knee arthroplasty owing to low sensitivity and negative predictive value, ${ }^{23,24}$ the utility in necrotizing soft tissue infection with a higher expected concentration of organisms requires further study.

Despite the potential for molecular testing to detect more organisms than would have been isolated by traditional culture methods, it has been suggested that a larger proportion of necrotizing soft tissue infection cases are monomicrobial than in prior years. ${ }^{25,26}$ Polymicrobial infections were substantially less common in this study than has been reported in the literature ( $29 \mathrm{vs}>70 \%$ ), a difference that could be attributed to the predominance of extremity lesions associated with more aggressive monomicrobial infections, which may have resulted in more frequent referrals to Brigham and Women's
Hospital. ${ }^{27}$ Irrespective of other trends, Group A streptococci remains the most common individual isolate both in the literature and in this study. ${ }^{28}$

The results of this study may overestimate the frequency of discrepancies between frozen and permanent sections, owing to histologic artifacts on some of the older slides that preclude optimal review. A small number of permanent sections lacked findings seen on frozen section slides owing to exhaustion of diagnostic tissue, but were not considered to be clinically relevant since they would be unlikely to change patient care. Substantial variation between pathologists was observed with independent review of the slides. As expected, categories with multiple diagnostic options such as neutrophils and necrosis had lower rates of agreement, including several cases with three different scores, as indicated by kappa statistics in the fair to moderate range. In contrast, the presence of bacteria and fibrin thrombi had the highest rates of concordance, possibly owing to the increased objectivity of evaluation of these features, with kappa statistics in the moderate to substantial range. Surprisingly, karyorrhexis and fibrin were often discordant, owing to frozen section artifacts and different personal thresholds for making the diagnosis. Each pair of pathologists agreed at a similar rate (69-75\%), indicating no single pathologist was markedly different from the group.

The most efficient method to identify patients with necrotizing soft tissue infection prior to surgical intervention has yet to be determined. A major limitation of this study, therefore, was the lack of a widely-accepted gold standard for comparison, which instead relied heavily on post-surgical diagnosis and clinical course. Clinical signs and symptoms, laboratory values, and imaging findings may be useful in 
Table 2 Correlation of culture results with histological evidence of bacteria

Histological evidence of bacteria

Culture results (necrotizing soft tissue infection-positive cases)

\begin{tabular}{ccc}
\hline Present $(\mathrm{n}=23)$ & Absent $(\mathrm{n}=40)$ & Total $(\mathrm{n}=63)$ \\
7 & 11 & 18 \\
9 & 6 & 15 \\
0 & 2 & 2 \\
1 & 0 & 1 \\
2 & 4 & 6 \\
0 & 4 & 4 \\
1 & 1 & 2 \\
0 & 2 & 2 \\
1 & 1 & 2 \\
1 & 1 & 2 \\
0 & 1 & 1 \\
1 & 7 & 8
\end{tabular}

Table 3 Concordance between individual pathologists in evaluation of frozen section slides

\begin{tabular}{lccccccc}
\hline & \multicolumn{5}{c}{ Histological feature } \\
\cline { 2 - 7 } Pathologists & Neutrophils & Necrosis & Thrombi & Bacteria & Karyorrhexis & Fibrin & Overall \\
\hline A+B+C & 51.8 & 49.4 & 77.1 & 73.5 & 50.6 & 59.0 & 60.3 \\
Kappa & 0.39 & 0.41 & 0.70 & 0.43 & 0.32 & 0.45 & 71.7 \\
A+B & 63.9 & 59.6 & 92.2 & 86.1 & 60.2 & 75.3 & 80.1 \\
A+C & 69.3 & 61.4 & 81.9 & 81.3 & 64.5 & 68.7 \\
B+C & 58.4 & 61.4 & 79.5 & 78.9 & 68.6 \\
\hline
\end{tabular}

Values represent percentage agreement or Kappa statistics calculated for interobserver variability between three pathologists.

some settings. The bedside finger test with frozen sections has been suggested to provide a benefit. Interestingly, the number of frozen sections per year has decreased gradually over time since a peak in 2002, and is likely to be a combination of ancillary diagnostics and surgeon preference. The goal of this study was to determine the utility of frozen section analysis, as an independent test, in the diagnosis of necrotizing soft tissue infection. This study did not include cases without frozen sections, precluding a comparison of outcomes or benefits, and was also limited by a lack of data on the correlation of biopsy results with outcomes or clinical decision making. As expected, frozen sections showed a greater number of neutrophils, increased extent of necrosis, and higher frequency of bacteria, karyorrhexis, and fibrin in necrotizing soft tissue infection-positive cases than negative, although no combination of histological findings was both sensitive and specific for the diagnosis of necrotizing soft tissue infection. Discordance between frozen and permanent sections is common, with at least one histological feature identified in 18/63 (29\%) necrotizing soft tissue infection cases. We also found substantial interobserver variability among pathologists in frozen section analysis (28\% overall discordance). These results support the current Infectious Disease Society of America and World Society of Emergency Surgery guidelines, which do not recommend delaying surgical treatment for frozen section biopsy results. ${ }^{29,30}$ Owing to limited sensitivity and specificity, caution should be used when utilizing frozen sections for the diagnosis of necrotizing soft tissue infection, which may result in false negatives and less aggressive treatment. In grossly positive cases, frozen sections are unlikely to add additional diagnostic information, but may delay definitive diagnosis. Further study is required to determine whether frozen section results could be effectively supplemented by STAT intraoperative Gram stains or combined with other clinical, laboratory, or radiological findings to improve diagnostics and outcomes in difficult cases in which initial surgical exploration has also failed to arrive at a definitive diagnosis.

\section{Acknowledgments}

We thank Manuel Castillo Angeles for assistance in performing statistical analyses. This work was presented in part at the United States and Canadian Association of Pathologists Annual Meeting in San Antonio, TX, 4-10 March 2017.

\section{Disclosure/conflict of interest}

The authors declare no conflict of interest. 


\section{References}

1 Arif N, Yousfi S, Vinnard C. Deaths from necrotizing fasciitis in the United States, 2003-2013. Epidemiol Infect 2016;144:1338-1344.

2 Hakkarainen TW, Kopari NM, Pham TN, et al. Necrotizing soft tissue infections: review and current concepts in treatment, systems of care, and outcomes. Curr Probl Surg 2014;51:344-362.

3 Misiakos EP, Bagias G, Patapis P, et al. Current concepts in the management of necrotizing fasciitis. Front Surg 2014;1:36.

4 Faraklas I, Yang D, Eggerstedt M, et al. A multi-center review of care patterns and outcomes in necrotizing soft tissue infections. Surg Infect (Larchmt) 2016;17: 773-778.

5 Psoinos CM, Flahive JM, Shaw JJ, et al. Contemporary trends in necrotizing soft-tissue infections in the United States. Surgery 2013;153:819-827.

6 Elliott D, Kufera JA, Myers RA. The microbiology of necrotizing soft tissue infections. Am J Surg 2000;179: 361-366.

7 Angoules AG, Kontakis G, Drakoulakis E, et al. Necrotising fasciitis of upper and lower limb: a systematic review. Injury 2007;38:S19-S26.

8 Wang YS, Wong CH, Tay YK. Staging of necrotizing fasciitis based on the evolving cutaneous features. Int J Dermatol 2007;46:1036-1041.

9 Keung EZ, Liu X, Nuzhad A, et al. Immunocompromised status in patients with necrotizing soft-tissue infection. JAMA Surg 2013;148:419-426.

10 Swain RA, Hatcher JC, Azadian BS, et al. A five-year review of necrotising fasciitis in a tertiary referral unit. Ann R Coll Surg Engl 2013;95:57-60.

11 Wong $\mathrm{CH}$, Khin LW, Heng KS, et al. The LRINEC (Laboratory Risk Indicator for Necrotizing Fasciitis) score: a tool for distinguishing necrotizing fasciitis from other soft tissue infections. Crit Care Med 2004;32:1535-1541.

12 Burner E, Henderson SO, Burke G, et al. Inadequate sensitivity of laboratory risk indicator to rule out necrotizing fasciitis in the emergency department. West J Emerg Med 2016;17:333-336.

13 Chaudhry AA, Baker KS, Gould ES, et al. Necrotizing fasciitis and its mimics: what radiologists need to know. AJR Am J Roentgenol 2015;204:128-139.

14 Malghem J, Lecouvet FE, Omoumi P, et al. Necrotizing fasciitis: contribution and limitations of diagnostic imaging. Joint Bone Spine 2013;80:146-154.

15 Andreasen TJ, Green SD, Childers BJ. Massive infectious soft-tissue injury: diagnosis and management of necrotizing fasciitis and purpura fulminans. Plast Reconstr Surg 2001;107:1025-1035.
16 Anaya DA, Dellinger EP. Necrotizing soft-tissue infection: diagnosis and management. Clin Infect Dis 2007;44:705-710.

17 Stamenkovic I, Lew PD. Early recognition of potentially fatal necrotizing fasciitis. The use of frozen-section biopsy. N Engl J Med 1984;310:1689-1693.

18 Hietbrink F, Bode LG, Riddez L, et al. Triple diagnostics for early detection of ambivalent necrotizing fasciitis. World J Emerg Surg 2016;11:51.

19 Majeski J, Majeski E. Necrotizing fasciitis: improved survival with early recognition by tissue biopsy and aggressive surgical treatment. South Med J 1997;90: 1065-1068.

20 Stegeman SA, Nijhuis I, van Leeuwen AM, et al. The value of frozen section biopsy in diagnosing necrotizing fasciitis: proposal of a new grading system. J Tissue Viability 2012;21:13-16.

21 White VA, Trotter MJ. Intraoperative consultation/final diagnosis correlation: relationship to tissue type and pathologic process. Arch Pathol Lab Med 2008;132: 29-36.

22 Howanitz PJ, Hoffman GG, Zarbo RJ. The accuracy of frozen-section diagnoses in 34 hospitals. Arch Pathol Lab Med 1990;114:355-359.

23 Shifflett GD, Nwachukwu BU, Bjerke-Kroll BT, et al. The value of intraoperative Gram stain in revision spine surgery. Spine J 2015;15:2198-2205.

24 Morgan PM, Sharkey P, Ghanem E, et al. The value of intraoperative Gram stain in revision total knee arthroplasty. J Bone Joint Surg Am 2009;91:2124-2129.

25 Tsitsilonis S, Druschel C, Wichlas F, et al. Necrotizing fasciitis: is the bacterial spectrum changing? Langenbecks Arch Surg 2013;398:153-159.

26 Rudkjobing VB, Thomsen TR, Xu Y, et al. Comparing culture and molecular methods for the identification of microorganisms involved in necrotizing soft tissue infections. BMC Infect Dis 2016;16:652.

27 Childers BJ, Potyondy LD, Nachreiner R, et al. Necrotizing fasciitis: a fourteen-year retrospective study of 163 consecutive patients. Am Surg 2002;68:109-116.

28 Glass GE, Sheil F, Ruston JC, et al. Necrotising soft tissue infection in a UK metropolitan population. Ann R Coll Surg Engl 2015;97:46-51.

29 Stevens DL, Bisno AL, Chambers HF, et al. Practice guidelines for the diagnosis and management of skin and soft tissue infections: 2014 update by the Infectious Diseases Society of America. Clin Infect Dis 2014;59: e10-e52.

30 Sartelli M, Malangoni MA, May AK, et al. World Society of Emergency Surgery (WSES) guidelines for management of skin and soft tissue infections. World J Emerg Surg 2014;9:57. 